\title{
Eight decades of asymptomatic Morgagni Hernia
}

Dear Sir,

Morgagni hernia is a rare type of congenital hernia which occurs due to a defect in the antero-medial part of the diaphragm wherein the abdominal contents herniate into the thorax. ${ }^{1}$ The condition was first described by Giovanni Battista Morgagni in the year 1761. ${ }^{2}$ Patients are usually asymptomatic and the diagnosis is incidental during routine chest imaging. The management is by surgical correction of the diaphragmatic defect.

An 82 year old lady presented to the Medicine OPD with complaints of fever and burning micturation. She is not a known case of any comorbid condition and was not on any regular medications. There was no history of any previous hospitalization. On examination, she was overweight (BMI $28.4 \mathrm{~kg} / \mathrm{m}^{2}$ ). Her vitals were stable. Systemic examination revealed decreased air entry in the right infraaxillary and mammary areas. Her blood investigations showed leucocytosis $(13,400$ cells $/ \mathrm{cmm})$ with neutrophilia (N90 L10). Urine microscopy showed plenty of pus cells. Her renal and liver parameters, electrolytes, HbA1c and ECG were normal. Her chest $\mathrm{X}$ ray showed a homogenous mass occupying the right lower zone (Figure 1). CT thorax was suggestive of Morgagni hernia with omental herniation (Figure 2). Her urine culture grew E.coli and she was treated with intravenous ceftriaxone (as per culture and sensitivity reports). She was advised surgical correction of her Morgagni hernia; but considering her age and the asymptomatic nature of the condition for the past 8 decades, the patient and her relatives were not willing for any intervention.

Morgagni hernia represents $3 \%$ of congenital diaphragmatic hernias, making them the rarest form. The defect is in the fusion of the costal arches with the septum transverses of the diaphragm. ${ }^{3}$ The herniation is usually seen on the right side of the diaphragm. Bilateral Morgagni hernia may occur; but left diaphragmatic herniation is rare because of the presence of the pericardial sac. Omentum, transverse colon, liver, small bowel and stomach are the usual contents of the hernia. About 50 to $70 \%$ of these patients are asymptomatic. The incidence is higher among females.

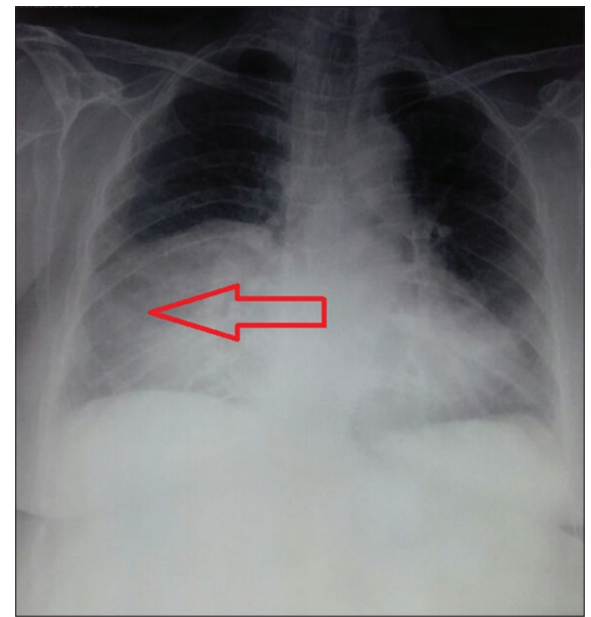

Figure 1: Chest Xray showing a homogenous mass occupying the right lower zone.

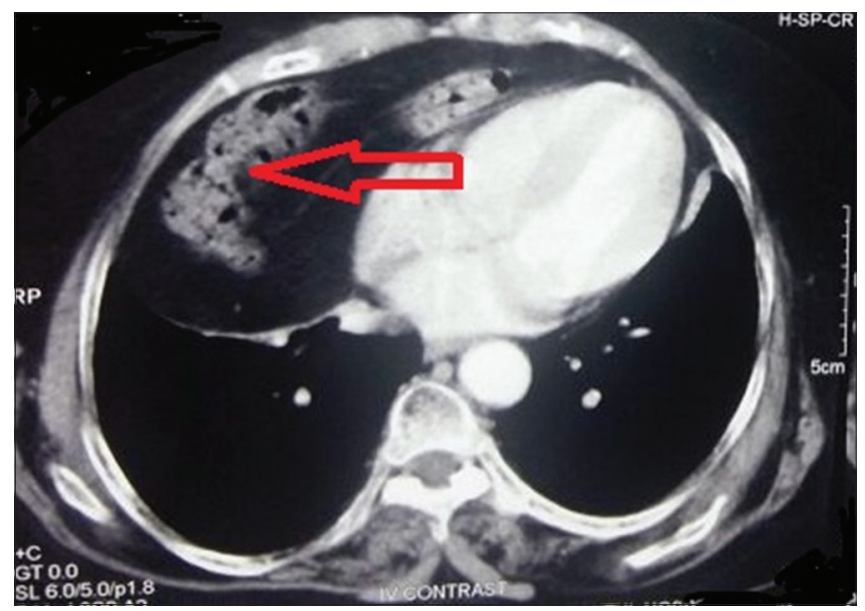

Figure 2: CT Thorax showing Morgagni hernia on the right side with omental herniation.

Obesity is considered as a risk factor. Though the condition is congenital, the patients are usually above 50 years of age at the time of presentation. ${ }^{4}$ 
Imaging modalities like chest or abdominal $\mathrm{X}$ ray, ultrasound, CT and MRI aids in the diagnosis of Morgagni hernia. ${ }^{1}$ The treatment is by surgical repair of the hernia, which may be done by transabdominal or transthoracic approach. Bilateral and complicated hernia sacs are repaired by transabdominal approach. However, this might be difficult in obese patients. ${ }^{3}$ Transthoracic approach, on the other hand, is an easier method of repair and holds better outcome. ${ }^{5}$ Laparoscopic and thoracoscopic repairs have also been done. ${ }^{4}$ Untreated cases may develop life threatening complications like obstruction and strangulation.

Our patient must be one the oldest reported asymptomatic Morgagni hernia with omental herniation in medical literature.

Key words: Morgagni hernia, Diaphragmatic hernia, Congenital hernia

Robin George Manappallil', Mele Chelakkoth Sabir ${ }^{2}$, Shameemu Rahman ${ }^{3}$
${ }^{1}$ Consultant, Department of Internal Medicine, Baby Memorial Hospital, Calicut, Kerala, India, ${ }^{2}$ Consultant, Department of Pulmonary Medicine, Baby Memorial Hospital, Calicut, Kerala, India ${ }^{3}$ Consultant, Department of Internal Medicine, Santhi Hospital, Calicut, Kerala, India

Address for correspondence: Dr. Robin George Manappallil, Consultant Physician, Department of Internal Medicine, Baby Memorial Hospital, Calicut, Kerala, India 673004. Mobile: +91-8547753396. E-mail: drrobingeorgempl@gmail.com

\section{REFERENCES}

1. Eren S and Ciris F. Diaphragmatic hernia: diagnostic approaches with review of the literature. Eur J Radiol 54448-459.

2. Guttman FM and Laberge JM. Congenital diaphragmatic hernia. In: Nyhous LM, Condon RE (eds) Hernia. JB Lippincott Company, Philadelphia, p 694.

3. Loong TP and Kocher HM. Clinical presentation and operative repair of hernia of Morgagni. Postgrad Med J; 8141-44.

4. Minneci PC, Deans KJ, Kim P and Mathisen DJ. Foramen of Morgagni hernia: changes in diagnosis and treatment. Ann Thorac Surg 771956-1959.

5. Kiliç D, Nadir A, Döner E, Kavukçu S, Akal M, Ozdemir N, et al. Transthoracic approach in surgical management of Morgagni hernia. Eur J Cardiothorac Surg 201016-1019.

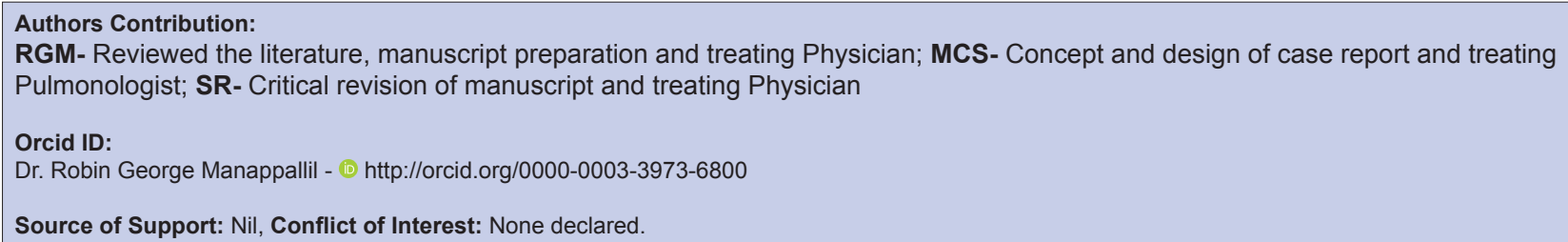

